\title{
Metabolic heat in microbial conflict and cooperation
}

\begin{abstract}
Steven A. Frank*
Many microbes live in habitats below their optimum temperature. Retention of metabolic heat by aggregation or insulation would boost growth. Generation of excess metabolic heat may also provide benefit. A cell that makes excess metabolic heat pays the cost of production, whereas the benefit may be shared by neighbors within a zone of local heat capture. Metabolic heat as a shareable public good raises interesting questions about conflict and cooperation of heat production and capture. Metabolic heat may also be deployed as a weapon. Species with greater thermotolerance gain by raising local temperature to outcompete less thermotolerant taxa. Metabolic heat may provide defense against bacteriophage attack, by analogy with fever in vertebrates. This article outlines the theory of metabolic heat in microbial conflict and cooperation, presenting several predictions for future study.
\end{abstract}

Keywords: Thermoregulation, microbial metabolism, overflow metabolism, biofilms, public goods, social evolution, ecological competition, fever, bacteriophage defense

\section{Introduction}

Metabolic heat may play an important role in microbial conflict and cooperation. On the conflict side, microbes often differ in their temperature optima ${ }^{1}$. A microbe that raises the local temperature closer to its own optimum gains a growth advantage over relatively thermophobic competitors.

On the cooperative side, aggregates may retain metabolic heat and gain a growth rate advantage ${ }^{2}$. Internal cells in an aggregate potentially benefit by generating excess heat, the energy cost reducing their own growth but stimulating faster growth among neighboring genetic relatives at the periphery. Cool environments with slow heat dissipation favor cooperative thermogenesis.

The theory builds on three assumptions. First, temperature influences fitness. Second, individual and group traits can modulate heat production and heat flow. Third, heat flow affects local temperature and thus the fitness of neighbors.

I discuss each assumption. I then turn to predictions.

\footnotetext{
* Department of Ecology and Evolutionary Biology, University of California, Irvine, CA 92697-2525, USA

web: https://stevefrank.org
}

\section{Fitness consequences: competition}

Competition requires that taxa differ in their temperature response. Types with relatively higher temperature optima or greater tolerance to heat can potentially gain an advantage by warming the local environment.

Alster et al. $^{1}$ quantified temperature response in terms of thermodynamic variables that determine reaction rates. Thermodynamic measures of reaction rates are not direct measures of fitness. However, differences in the temperature sensitivity of metabolic processes likely influence temperature differences in growth rate and metabolic efficiency.

Alster et al. $^{1}$ focused on three variables. The optimum temperature maximizes reaction rate. The heat capacity determines the breadth of the temperature response curve, with greater heat capacity corresponding to a broader temperature response curve and less overall sensitivity to temperature variability. Maximum temperature sensitivity defines the point at which the reaction rate changes most rapidly with respect to temperature.

Literature meta-analysis yielded 353 response curves across diverse microbial groups ${ }^{1}$. The distribution of optimum temperature is approximately a right-skewed Gaussian shape with a mean and standard deviation of $29.4 \pm 10.1^{\circ} \mathrm{C}$. Heat capacity and 
maximum temperature sensitivity also vary widely.

Many laboratory studies have measured fitness at different temperatures ${ }^{3-6}$. Growing microbes at higher or lower temperatures often causes an evolutionary shift in temperature response, demonstrating lability of thermotolerance. Protein thermosensitivity likely explains a significant part of the variability in the temperature response curves of taxa ${ }^{7,8}$.

Overall, variation in observed temperature response suggests wide scope for using metabolic heat as a competitive weapon.

\section{Fitness consequences: cooperation}

The potential for cooperation requires that microbes sometimes live in habitats below their optimum temperature. When below the optimum, excess heat can be a shareable public good that is costly to produce and potentially beneficial to neighbors. I found only one study of metabolic heat used to raise local temperature for colony benefit ${ }^{2}$.

Several examples suggest that increased local temperature could be advantageous in cold environments.

Some organisms use dark pigmentation to raise cellular temperature. Cordero et al. ${ }^{9}$ showed that pigmentation increases in yeast with latitude. That increase suggests that high latitude taxa gain from raising their temperature above the ambient level. When grown in the lab at $4^{\circ} \mathrm{C}$ under light, melanized Crytococcus neoformans gained a growth advantage relative to nonmelanized taxa, but at $23^{\circ} \mathrm{C}$ the melanized form suffered increased thermal stress.

Most of the biosphere is permanently cold, including alpine, arctic, and oceanic habitats ${ }^{10}$. Coldadapted microbes ${ }^{11}$ occupy these habitats down to about $-20^{\circ} \mathrm{C}$. Estimates suggest counts of approximately $10^{5}$ and $10^{6}$ cells $\mathrm{ml}^{-1}$ in Arctic ice pack and Antarctic sea ice, respectively ${ }^{12}$. Smaller counts have been observed in deep ice cores ${ }^{13}$.

Among taxa that could be cultured ${ }^{10}$, most isolates from cold habitats survived or grew at cold temperatures but reproduced most quickly at $20-25^{\circ} \mathrm{C}$. Only a few isolates grew fastest at cool temperatures of $10-15^{\circ} \mathrm{C}$. Thus, the capture and sharing of local metabolic heat may be particularly valuable in cold habitats.

\section{Individual and group traits}

Individuals may contribute heat by excess thermogenesis. Groups may retain heat by aggregation and by insulation.

Cellular aggregation is perhaps the simplest trait. I did not find studies of microbes that consider individual and group traits in terms of conflicting and cooperative aspects of thermoregulation. The closest analogy to my argument comes from huddling behavior in birds and mammals to retain heat.

Haig ${ }^{14,15}$ noted that heat is a public good in a vertebrate huddle. Heat generators pay the cost of production. The benefit is shared by all neighbors. Individuals can exploit warm neighbors by reducing their own heating budget. In broods, siblings and parents have various conflicting and cooperative interests with regard to heat.

Familial conflicts over physiological traits often associate with genomic imprinting in mammals ${ }^{16}$. Several imprinted genes in mice and humans influence thermogenesis and follow the common pattern for familial conflict ${ }^{14,17}$.

In microbes, aggregation by intercellular adhesion occurs widely. Cells may also aggregate by surface attachment and by active movement toward groups. Many possible costs and benefits of cellular aggregation occur ${ }^{18-22}$. However, heat in microbial aggregates has not been widely discussed.

In addition to aggregation, groups may also retain heat by secreting extracellular insulation. The idea that extracellular secretions function as insulation for microbial thermoregulation has not been widely discussed.

Biofilms combine aggregation and insulation. Apart from the one study mentioned above noting that aggregates may beneficially raise their temperature $^{2}$, I did not find discussion of increased temperature as an adaptive benefit of biofilms. The idea has likely been mentioned, but is not widely considered.

Heat may be used as a weapon against relatively thermophobic competitors or invaders. I did not find any literature on microbes that use metabolic heat as a weapon or as a defense against invading bacterio- 
phage.

Fever in vertebrates ${ }^{23}$ and social insects ${ }^{24}$ provides an analogy. Those organisms sometimes raise their temperature to control microbial invaders. Observations suggest that Japanese honey bees surround invading Asian giant hornets (Vespa mandarinia) and generate excess heat and $\mathrm{CO}_{2}$ to kill their relatively thermophobic enemy ${ }^{25}$.

These various benefits of excess heat require that some individuals in the group spend metabolic energy on heat production. Microbes have metabolic flux pathways that seem designed to dissipate excess ATP without driving any anabolic processes. Those energy spilling reactions release significant heat, sometimes associated with a futile cycle of proton flux through the cell membrane ${ }^{26}$.

A few possible functions for futile cycles have been mentioned, such as correcting thermodynamic imbalance $^{27}$. However, the Tabata et al. article ${ }^{2}$ is the only one I found that suggests heat generation may itself be a benefit.

If free energy limits reproduction, then individuals that generate excess heat may be reducing their own reproduction in favor of the group-level benefit shared by neighbors. With heat as a public good ${ }^{28}$, competitive nonproducers could gain a growth advantage against cooperative heat producers.

In dense, energy-rich environments that dissipate heat relatively slowly, metabolic heat can raise local temperatures beyond the optimum for growth. Excreting catabolic intermediates such as lactate, acetate or ethanol may reduce heat production to keep temperatures below stressful levels. Protection against overheating provides an alternative explanation for the puzzle of overflow metabolism ${ }^{29-31}$.

\section{Heat flow and spatial scale}

Metabolic heat can alter local temperature and potentially be important in conflict and cooperation. However, local heat must dissipate sufficiently slowly to play an important role. Here, "slowly" means the scaling of heat dissipation relative to the rate of other processes.

For example, does excess heat dissipate slowly enough that it can raise the rates of metabolic re- actions and the growth rate of neighbors? Can excess heat be sufficiently concentrated to be used as a weapon that reduces the growth rate of relatively thermophobic competitors? What aspects of cellular aggregation and biofilm properties retain heat sufficiently to raise growth rate? How do changes in heat flow trade off against changes in the flow of other resources? How do larger-scale biophysical aspects of a habitat interact with smaller-scale intercellular processes to affect overall heat conductance?

Habitats vary in thermal properties. For example, water content and particle size significantly influence heat flow in soils ${ }^{32}$. Water absorbs and dissipates heat more rapidly than does air. Convective flow may often dominate in the movement of heat. Still habitats may therefore be better candidates for local concentration of heat.

Rates of small-scale heat dissipation have typically not been focal aspects of microbial studies. Microcalorimetric methods provide a way to measure heat at small spatial scales ${ }^{33}$. Future improvements in technology will likely enhance spatial resolution, which may improve the tracking of heat flow over the spatial scales at which conflict and cooperation play out.

\section{Predictions}

Several broad predictions summarize key points and potential applications.

Relatively cold habitats more strongly favor excess metabolic heat to raise local temperature.

Habitats that dissipate heat more slowly favor the benefits of local heat production more strongly.

Cellular aggregation and extracellular insulation to retain local heat are more strongly favored as the growth rate benefits from heat become more valuable competitively.

The more genetically distinct cells are in an aggregation, the more likely that some cells do not contribute to costly heat production (the public goods dilemma).

In cellular aggregations, internal cells are more likely to generate excess heat because their heat production is protected by greater insulation than peripheral cells. 
Species with broader thermal tolerance and higher optimum temperature are more likely to use local heat as a competitive weapon.

In cool insulated environments, thermal response curves may evolve by competitive game-like dynamics, increasing heat tolerance and success at higher temperatures.

Species with broader thermal tolerance are more likely to use excess heat generation as a defensive fever response against invading bacteriophage.

The benefit of competitive and defensive heat generation may often increase with the number of cells that cooperate to create a thermal weapon, suggesting a link to quorum sensing.

The benefit of competitive and defensive heat generation rises with the tendency of the aggressors to surround their foe.

In dense environments that tend to overheat, microbes may secrete catabolic intermediates in overflow metabolism to reduce heat generation.

In summary, heat plays a primary role in the rate processes of life. Various individual and group traits of heat generation, cellular aggregation, and extracellular insulation may influence aspects of conflict and cooperation in microbial communities.

\section{Acknowledgments}

The Donald Bren Foundation and NSF grant DEB1939423 support my research.

\section{References}

1. Alster, C. J., Weller, Z. D. \& von Fischer, J. C. A meta-analysis of temperature sensitivity as a microbial trait. Global Change Biology 24, 42114224 (2018).

2. Tabata, K. et al. Measurement of soil bacterial colony temperatures and isolation of a high heatproducing bacterium. BMC Microbiology 13, 56 (2013).

3. Bennett, A. F. \& Lenski, R. E. An experimental test of evolutionary trade-offs during temperature adaptation. Proceedings of the National Academy of Sciences 104, 8649-8654 (2007).

4. Chen, P. \& Shakhnovich, E. I. Thermal adaptation of viruses and bacteria. Biophysical Journal 98, 1109-1118 (2010).

5. Caspeta, L. \& Nielsen, J. Thermotolerant yeast strains adapted by laboratory evolution show trade-off at ancestral temperatures and preadaptation to other stresses. mBio 6, e00431-15 (2015).

6. Yung, C.-M. et al. Thermally adaptive tradeoffs in closely related marine bacterial strains. Environmental Microbiology 17, 2421-2429 (2015).

7. Ghosh, K. \& Dill, K. Cellular proteomes have broad distributions of protein stability. Biophysical Journal 99, 3996-4002 (2010).

8. Chen, K. et al. Thermosensitivity of growth is determined by chaperone-mediated proteome reallocation. Proceedings of the National Academy of Sciences 114, 11548-11553 (2017).

9. Cordero, R. J. et al. Impact of yeast pigmentation on heat capture and latitudinal distribution. Current Biology 28, 2657-2664 (2018).

10. Rodrigues, D. F. \& Tiedje, J. M. Coping with our cold planet. Appl. Environ. Microbiol. 74, 16771686 (2008).

11. D’Amico, S., Collins, T., Marx, J.-C., Feller, G. \& Gerday, C. Psychrophilic microorganisms: challenges for life. EMBO Reports 7, 385-389 (2006).

12. Brinkmeyer, R. et al. Diversity and structure of bacterial communities in arctic versus antarctic pack ice. Appl. Environ. Microbiol. 69, 66106619 (2003).

13. Price, P. B. \& Sowers, T. Temperature dependence of metabolic rates for microbial growth, maintenance, and survival. Proceedings of the National Academy of Sciences 101, 4631-4636 (2004).

14. Haig, D. Huddling: brown fat, genomic imprinting and the warm inner glow. Current Biology 18, R172-R174 (2008).

15. Haig, D. The huddler's dilemma: a cold shoulder or a warm inner glow. In Székely, T., Moore, A. J. \& Komdeur, J. (eds.) Social Behaviour: Genes, Ecology and Evolution, 107-109 (Cambridge University Press, 2010).

16. Haig, D. Transfers and transitions: parentoffspring conflict, genomic imprinting, and the evolution of human life history. Proceeding of the National Academy of Sciences USA 107, 1731- 
1735 (2010).

17. Crespi, B. J. Why and how imprinted genes drive fetal programming. Frontiers in Endocrinology 10, 940 (2020). URL https://www. frontiersin.org/article/ 10.3389/fendo. 2019.00940.

18. Grosberg, R. K. \& Strathmann, R. R. The evolution of multicellularity: a minor major transition? Annu. Rev. Ecol. Evol. Syst. 38, 621-654 (2007).

19. Koschwanez, J. H., Foster, K. R. \& Murray, A. W. Sucrose utilization in budding yeast as a model for the origin of undifferentiated multicellularity. PLoS Biology 9 (2011).

20. Smith, J., Queller, D. C. \& Strassmann, J. E. Fruiting bodies of the social amoeba dictyostelium discoideum increase spore transport by Drosophila. BMC Evolutionary Biology 14, 105 (2014).

21. Ratcliff, W. C., Fankhauser, J. D., Rogers, D. W., Greig, D. \& Travisano, M. Origins of multicellular evolvability in snowflake yeast. Nature Communications 6, 1-9 (2015).

22. Kuzdzal-Fick, J. J., Chen, L. \& Balázsi, G. Disadvantages and benefits of evolved unicellularity versus multicellularity in budding yeast. Ecology and Evolution 9, 8509-8523 (2019).

23. Evans, S. S., Repasky, E. A. \& Fisher, D. T. Fever and the thermal regulation of immunity: the immune system feels the heat. Nature Reviews Immunology 15, 335-349 (2015).

24. Starks, P. T., Blackie, C. A. \& Seeley, T. D. Fever in honeybee colonies. Naturwissenschaften $\mathbf{8 7}$, 229-231 (2000).

25. Sugahara, M. \& Sakamoto, F. Heat and car- bon dioxide generated by honeybees jointly act to kill hornets. Naturwissenschaften 96, 11331136 (2009). URL https://doi.org/10.1007/ s00114-009-0575-0.

26. Russell, J. B. The energy spilling reactions of bacteria and other organisms. Journal of Molecular Microbiology and Biotechnology 13, 1-11 (2007).

27. Von Stockar, U. \& Liu, J.-S. Does microbial life always feed on negative entropy? thermodynamic analysis of microbial growth. Biochimica et Biophysica Acta (BBA)-Bioenergetics 1412, 191-211 (1999).

28. West, S. A., Diggle, S. P., Buckling, A., Gardner, A. \& Griffin, A. S. The social lives of microbes. Annual Review of Ecology and Systematics 38, 5377 (2007).

29. Warburg, O. On the origin of cancer cells. Science 123, 309-314 (1956).

30. Postma, E., Verduyn, C., Scheffers, W. A. \& Van Dijken, J. P. Enzymic analysis of the crabtree effect in glucose-limited chemostat cultures of Saccharomyces cerevisiae. Appl. Environ. Microbiol. 55, 468-477 (1989).

31. Wolfe, A. J. The acetate switch. Microbiol. Mol. Biol. Rev. 69, 12-50 (2005).

32. Usowicz, B., Lipiec, J., Usowicz, J. B. \& Marczewski, W. Effects of aggregate size on soil thermal conductivity: Comparison of measured and model-predicted data. International Journal of Heat and Mass Transfer 57, 536-541 (2013).

33. Braissant, O., Wirz, D., Göpfert, B. \& Daniels, A. U. Use of isothermal microcalorimetry to monitor microbial activities. FEMS Microbiology Letters 303, 1-8 (2010). 